\title{
Endovascular Revascularization of a Symptomatic Common Carotid Artery Occlusion
}

\author{
Julie Kromm, Tim E. Darsaut, Khurshid Khan, Robert Ashforth, Esseddeeg Ghrooda
}

\begin{abstract}
Although the common carotid artery is the second most common site for extracranial carotid artery stenosis, complete symptomatic occlusion in the absence of devastating stroke is rare. We present a case of complete common carotid artery occlusion failing medical management and requiring endovascular intervention. The clinical presentation, diagnostic investigations, and management of complete carotid artery occlusions are discussed.
\end{abstract}

RÉSUMÉ: Revascularisation endovasculaire à la suite de l'occlusion symptomatique de l'artère carotide commune. Bien que l'artère carotide commune soit le deuxième vaisseau de l'appareil circulatoire où se produit le plus fréquemment une sténose extracrânienne, une occlusion complète et symptomatique demeure rare en l'absence d'un AVC dévastateur. Nous voulons présenter un cas d'occlusion complète de l'artère carotide commune dont le traitement médical a échoué et qui a nécessité une intervention de nature endovasculaire. Nous entendons aussi présenter sur le plan clinique des cas d'occlusion complète de l'artère carotide mais aussi les examens diagnostic et les traitements s'y rapportant.

Keywords: Angioplasty, Carotid artery disease, Cerebrovascular disease, Stroke

doi:10.1017/cjn.2016.412

Can J Neurol Sci. 2017; 44: 120-123

After the internal carotid, the common carotid artery (CCA) is the second most common site for extracranial carotid artery stenosis. ${ }^{1}$ Despite this, complete CCA occlusion remains relatively rare, occurring in $1 \%$ to $4 \%$ of patients with symptomatic cerebral vascular disease. ${ }^{1}$ Although the relationship between stenoses of the extracranial carotid and the function of the nervous system has been thoroughly studied over the past few decades, the majority of research has focused on stenosis of the internal carotid artery (ICA).

Successful intervention in the setting of CCA occlusion when required can be accomplished with medical management, with or without a surgical or endovascular procedure. ${ }^{1,2}$ Because of the rarity of CCA occlusion, little evidence is available to guide rational decision-making. We present a case of CCA occlusion occurring at a distance from the common carotid arterial origin, which supports the choice of endovascular management for this uncommon problem.

\section{Presentation}

A 58-year-old man with multiple vascular risk factors, presented with a four-day history of progressive right-sided hemiparesis, dysarthria, and aphasia. Examination revealed a National Institute of Health Stroke Scale score of 10 with mild expressive aphasia, dysarthria, right hemianopia, and right-sided hemiplegia.

While hospitalized, the aphasia remained fixed, whereas the hemianopsia and hemiplegia improved. Despite immediate optimization of medical management, including dual antiplatelet agents (aspirin $81 \mathrm{mg}$ and clopidogrel $75 \mathrm{mg}$ daily) and cessation of antihypertensive medications, the patient remained neurologically unstable for 10 days following admission, with a fluctuating motor examination; strength in the right upper and lower extremities decreased from $4+/ 5$ to $4-/ 5$ when he moved from a supine to an upright position.

\section{INVESTIGATIONS}

Computed tomography (CT) of the head showed evidence of a subacute left middle cerebral artery (MCA) infarct. Magnetic resonance imaging of the brain with diffusion-weighted sequences showed acute left hemisphere infarctions in a watershed distribution.

CT angiogram suggested a complete proximal left CCA occlusion with reconstitution of the left internal cerebral artery by the external cerebral artery (Figure 1A). CT perfusion scans completed on admission and 10 days later showed persistent mismatch with an increased mean transit time, decreased cerebral blood flow, and maintained cerebral blood volume, consistent with diffuse hypoperfusion of the left hemisphere compared with the right (Figure $1 \mathrm{~B}, \mathrm{C}$ ).

Transcranial Doppler on the day of admission showed reversed flow in the left ophthalmic artery and bidirectional flow in the anterior communicating artery. Transcranial Doppler also showed episodic reductions of left MCA blood flow ( $30 \%)$ compared with flow in the right MCA. Repeat transcranial Doppler studies

From the Division of Neurology, Department of Medicine, University of Alberta, Edmonton, Alberta, Canada (JK, KK); Department of Critical Care Medicine, University of Calgary, Calgary, Alberta, Canada (JK); Division of Neurosurgery, Department of Surgery, University of Alberta, Edmonton, Alberta, Canada (TED); Department of Radiology and Diagnostic Imaging, University of Alberta, Edmonton, Alberta, Canada (RA); Section of Neurology, Department of Medicine, University of Manitoba, Winnipeg, Manitoba, Canada (EG).

Received December 8, 2015. Final Revisions Submitted March 13, 2016. Date of Acceptance May 23, 2016.

Correspondence to: Julie Kromm, Division of Neurology, Department of Medicine, University of Alberta, 7-132 Clinical Science Building, Edmonton, Alberta T6G 2G3, Canada. Email: jkromm@ualberta.ca 

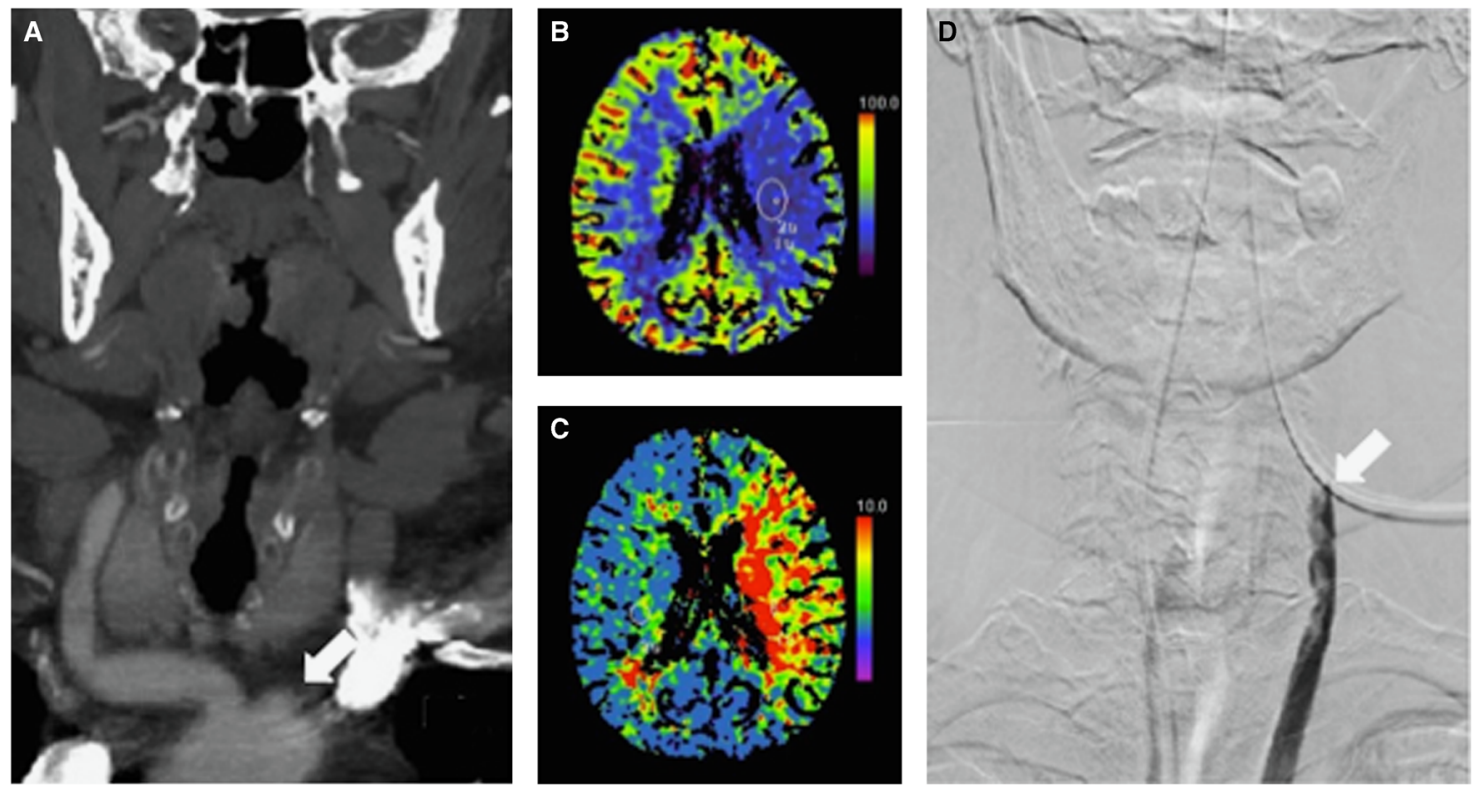

Figure 1: Preprocedure. CT angiography (A) showing complete occlusion (arrow) of the left common carotid artery. CT perfusion showing decreased cerebral blood flow $(B)$ and increased mean transit time $(C)$ in the left cerebral hemisphere, suggesting hypoperfusion. Digital subtraction angiography $(D)$ confirming left CCA complete occlusion (arrow).

on the fourth and fifth days following admission showed $>30 \%$ reduction in the left MCA flow velocity when the patient moved from a lying to sitting or standing position, in addition to sonographic evidence of multiple microemboli.

A catheter angiogram confirmed the complete occlusion of the left CCA approximately $3 \mathrm{~cm}$ from the aortic arch origin (Figure 1D).

Considering the positional symptomatology and radiologic evidence of cerebral hemodynamic compromise despite optimized medical management, some form of revascularization was deemed necessary, in spite of the apparent risks. Given the patient's body habitus (a short thick neck and barrel chest), coupled with significant medical comorbidities, an option avoiding a general anesthetic was deemed preferable, and the decision was made to first proceed with an endovascular attempt at revascularization.

\section{Endovascular Procedure}

Under conscious sedation and full heparinization, via an $8 \mathrm{~F}$ femoral sheath, an over-the-wire exchange was performed to place an $8 \mathrm{~F}$ concentric balloon catheter in the left CCA. The balloon catheter was inflated to achieve flow arrest, and the CCA occlusion was first transversed with a 0.035 Terumo wire, followed by a 4F Glidecath catheter. The diagnostic catheter was then exchanged over a $0.035 \mathrm{~J} 260 \mathrm{~cm}$ exchange wire, and an Ultra-thin Diamond $4 \mathrm{~mm}$ angioplasty balloon was used to partially dilate the stenotic segment. A FilterWire EZ embolic protection system was then navigated beyond the stenotic segment and deployed in the distal cervical carotid. Carotid stenting then proceeded as per usual routine, with a $7 \mathrm{~mm} \times 40 \mathrm{~mm}$ Wallstent placed across the stenotic segment, followed by a poststent balloon angioplasty with a $4 \mathrm{~mm} \times 20 \mathrm{~mm}$ Sterling Monorail balloon. Poststenting runs showed a patent stented left CCA and ICA without evidence of distal emboli (Figure 2A).

\section{Postprocedural Course}

No new neurological deficits were detected on examination, and a subsequent CT perfusion showed resolution of the left hemisphere relative hypoperfusion (Figure 2B, C). Repeat transcranial Doppler scans showed normalization of the direction of flow in the left MCA and ophthalmic and anterior communicating arteries with no evidence of further microemboli. The patient stabilized neurologically, with full resolution of his positional deficits, and was transferred to a rehabilitation facility on appropriate medical therapy including dual antiplatelet therapy for 6 weeks with eventual transition to aspirin $81 \mathrm{mg}$ daily. One month later, the patient was independent with a Modified Rankin Score and Barthel Index of 1 and 100, respectively. On follow-up 1 year later, the patient continues to do well with only subtle right hand weakness that is not functionally limiting. Follow-up CT angiography shows persistent patency of the stented left CCA and ICA.

\section{DISCUSSION}

Akin to the ICA, the CCA is commonly affected by atherosclerosis. Other potentially stenotic pathologies, including arterial dissection, arteritis, radiation vasculopathy, and cardioarterial emboli have also been implicated. ${ }^{1}$ In our patient, the etiology of occlusion was assumed to be atherosclerotic, given the indirect cross-sectional imaging evidence of associated microangiopathic changes and diffuse atherosclerosis in his other extracranial vessels as well other investigations proving noncontributory. His acute presentation was likely the result of sudden deterioration of a chronically stenotic lesion, with minimal time for collateral formation. The mechanism of cerebral ischemia appeared to be both hemodynamic and embolic, as previously reported. ${ }^{1}$

CCA occlusions can be immediately devastating or, alternatively, remain clinically silent. When symptoms are present 

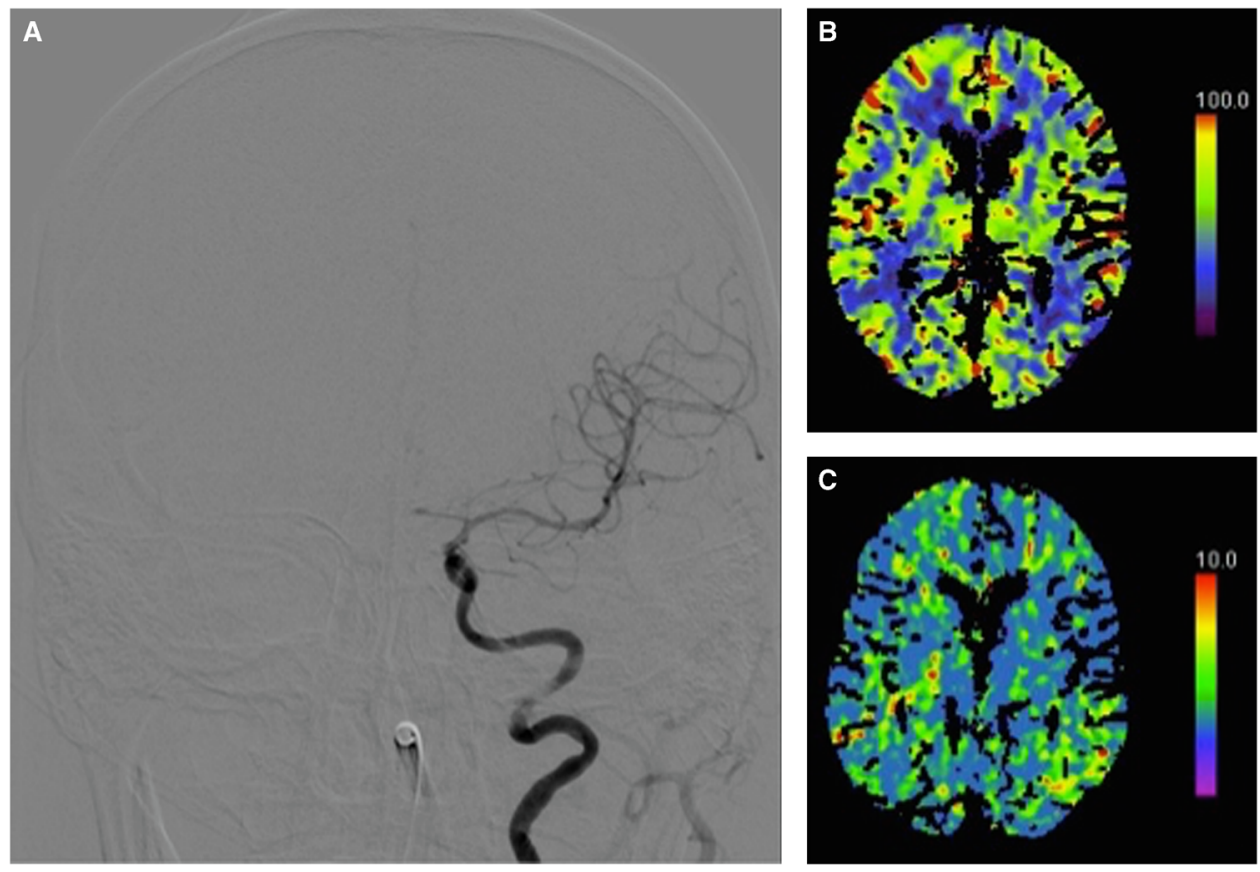

Figure 2: Postprocedure. Digital subtraction angiography (A) showing a patent stented left CCA and ICA. CT perfusion showing normalization of cerebral blood flow $(B)$ and mean transit time $(C)$.

they often resemble those of symptomatic ICA disease, with amaurosis fugax, transient ischemic attacks (limb-shaking events in the presence of hemodynamic compromise) or completed strokes. ${ }^{1}$ Although postural symptoms such as those seen with our patient are not a classic presentation for extracranial artery occlusion, they can certainly accompany patients with suddenly compromised hemodynamic reserve.

A full cerebral angiogram may be indicated to properly investigate complex cases, such as this patient with CCA occlusion, for treatment planning and to aid with ontologic classification, which is done according to the observed reconstitution of adjacent vasculature. ${ }^{1,3}$ With the advent of CT angiography and contrast-enhanced magnetic resonance angiography, we now have noninvasive diagnostic tests with sensitivity and specificity $>97 \%$ for detection of carotid artery occlusion. ${ }^{4}$ Doppler ultrasound is often physically limited by the inability to assess the deep ostial origin of the CCA, which can be affected in approximately $5 \%$ of patients. ${ }^{1}$ Because our patient had reconstitution of both the external and ICAs, the occlusion was classified as type 1A according to Riles' classification. ${ }^{1}$

The risk of ipsilateral ischemic stroke for patients with symptomatic carotid occlusion is thought to be in the range of $2 \%$ to $7 \%$ per year, even despite standard medical therapy. ${ }^{5}$ The risk may be higher (up to $30 \%$ per year) in patients with evidence of hemodynamic impairment as demonstrated by increased oxygen extraction fraction on positron emission tomography or reduced cerebrovascular reserve on acetazolamide-challenged singlephoton emission computed tomography. ${ }^{5}$ Although these studies were not completed in our case, CT perfusion, transcranial Doppler, and clinical examination all suggested hemodynamic impairment.

Medical management of extracranial atherosclerotic lesions is well-established in appropriate settings for both stenosis and occlusion. Surgical and especially interventional options for extracranial vascular disease are rapidly evolving, rendering it difficult to continue to manage patients with current level I evidence. ${ }^{6}$ Whether it is appropriate for clinicians to extrapolate ICA occlusion data to the setting of CCA occlusion remains unknown. The data available for CCA occlusion consist only of case reports and small series, which suggest that good outcomes are possible, although this is certainly affected by publication bias. ${ }^{2,7-12}$ Although many surgical revascularization options have been reported, patients who present difficult anatomic circumstances may have worse outcomes, particularly as a result of infrequently performed procedures. ${ }^{1}$ Although we acknowledge that blindly passing a guidewire through an occluded vessel that supplies viable brain is unlikely to become a routine procedure, it was believed to represent the best option given the circumstances. This procedure might equally well have dislodged a large thrombus and caused a massive stroke; we would recommend other practitioners facing a similar situation to be prepared with modern thrombectomy tools such as stent retrievers to prepare for that possibility. Finally, regardless of which type of procedure is performed, it remains important to maintain medical therapy following the procedure, to minimize the risk of the known short-term complications (cerebrovascular events, hyperperfusion syndrome, restenosis, or reocclusion.), and to optimize long term outcomes. $^{7-12}$

This case buttresses the sole existing report that successful endovascular management of an occluded CCA can be accomplished in a symptomatic patient with objective evidence of hemodynamic compromise. ${ }^{2}$ In this setting, surgical or endovascular management can be considered if the patient remains refractory to medical therapy. ${ }^{2,7-12}$ The site of occlusion in this case was at a distance from the common carotid arterial origin, which, in our opinion, rendered stenting more favorable than for 
endovascular management of an ostial lesion. Further studies are required before making recommendations regarding whether CCA occlusion patients should preferentially be managed with surgical, endovascular, or medical paradigms.

\section{Disclosures}

The authors do not have anything to disclose.

\section{REFERENCES}

1. Klonaris C, Kouvelos GN, Kafeza M, Koutsoumpelis A, Katsargyris A, Tsigris C. Common carotid artery occlusion treatment: revealing a gap in the current guidelines. Eur J Vasc Endovasc Surg. 2013;46:291-98.

2. Takagi T, Yoshimura S, Yamada K, Enomoto Y, Iwama T. Angioplasty and stenting of totally occluded common carotid artery at the chronic stage. Neurol. Med. Chir (Tokyo). 2010;50:998-1000.

3. Pritz MB. Usefulness of catheter angiography in the evaluation of common carotid artery origin occlusion. J Stroke Cerebrovasc Dis. 2008;17:42-6.

4. Saba L, Anzidei M, Sanfilippo R, et al. Imaging of the carotid artery. Atherosclerosis. 2013;220:294-309.

5. Grubb RL, Derdeyn CP, Fritsch SM, et al. Importance of hemodynamic factors in the prognosis of symptomatic carotid occlusion. JAMA. 1998;280:1055-60.
6. Liu ZJ, Fu WG, Guo ZY, Shen LG, Shi ZY, Li JH. Updated systematic review and meta-analysis of randomized clinical trials comparing carotid artery stenting and carotid endarterectomy in the treatment of carotid stenosis. Ann Vasc Surg. 2012; 26:576-90.

7. Thomas AJ, Gupta R, Tayal AH, Horowitz MB, Jovin TG. Stenting and angioplasty of the symptomatic chronically occluded carotid artery. AJNR Am J Neuroradiol. 2007;28:168-71.

8. Terada T, Yamaga H, Tsumoto T, Masuo O, Itakura T. Use of an embolic protection system during endovascular recanalization of a totally occluded cervical internal carotid artery at the chronic stage. J Neurosurg. 2005;102:558-64.

9. Terada T, Okada H, Nanto M, et al. Endovascular recanalization of the completely occluded internal carotid artery using a flow reversal system at the subacute to chronic stage. J Neurosurg. 2010;112:563-71.

10. Shojima M, Nemoto S, Morita A, et al. Protected endovascular revascularization of subacute and chronic total occlusion of the internal carotid artery. AJNR Am J Neuroradiol. 2010;3: 481-86.

11. Hauck EF, Ogilvy CS, Siddiqui AH, Hopkins LN, Levy EI. Direct endovascular recanalization of chronic carotid occlusion: should we do it? Case report. Neurosurgery. 2010;67:E1152-59.

12. Pintér L, Cagiannos C, Bakoyiannis CN, Kolvenbach R. Hybrid treatment of common carotid artery occlusion with ring-stripper endarterectomy plus stenting. J Vasc Surg. 2007;46:135-39. 